PROCEEDINGS OF THE

AMERICAN MATHEMATICAL SOCIETY

Volume 135, Number 3, March 2007, Pages 883-890

S 0002-9939(06)08521-2

Article electronically published on August 21, 2006

\title{
REALIZABILITY OF THE ADAMS-NOVIKOV SPECTRAL SEQUENCE FOR FORMAL $A$-MODULES
}

\author{
TYLER LAWSON
}

(Communicated by Paul Goerss)

\begin{abstract}
We show that the formal $A$-module Adams-Novikov spectral sequence of Ravenel does not naturally arise from a filtration on a map of spectra by examining the case $A=\mathbb{Z}[i]$. We also prove that when $A$ is the ring of integers in a nontrivial extension of $\mathbb{Q}_{p}$, the map $(L, W) \rightarrow\left(L_{A}, W_{A}\right)$ of Hopf algebroids, classifying formal groups and formal $A$-modules respectively, does not arise from compatible maps of $E_{\infty}$-ring spectra $(M U, M U \wedge M U) \rightarrow(R, S)$.
\end{abstract}

\section{INTRODUCTION}

The recent development of highly structured categories of spectra has led to questions about what kinds of algebro-geometric procedures can be imported into homotopy theory. In particular, it often leads to the hope that there might be "algebraic extensions" of the sphere spectrum that play the role of the ring of integers in a number field. For example, the notion of a Galois extension of a ring spectrum is defined in [6]. Unfortunately, the sphere spectrum has no Galois extensions unless one inverts a set of integers, roughly because Galois extensions cannot have ramified primes.

Additionally, Schwänzl, Vogt, and Waldhausen have shown that there exists no $A_{\infty}$-ring spectrum $R$ such that $\mathrm{HZ} \wedge R \simeq \mathrm{H} \mathbb{Z}[i]$ by making use of a calculation in topological Hochschild homology [7]. (Assuming $R$ is connective, it would necessarily have the homotopy type of $\mathbb{S} \vee \mathbb{S}$, with some $A_{\infty}$-structure imposed.)

This might suggest that another approach would be in order, based on different algebraic properties of the sphere spectrum. One such approach is through the Adams-Novikov spectral sequence. This spectral sequence computes the stable homotopy groups of the sphere and has an $E_{2}$-term given by Ext groups of the Hopf algebroid $\left(M U_{*}, M U_{*} M U\right)$. Due to the work of Quillen [4, it is possible to identify this $E_{2}$-term as Ext groups of the Hopf algebroid $(L, W)$ representing formal groups and strict isomorphisms between them.

Ravenel, in his article [5], defined the Adams-Novikov spectral sequence for formal $A$-modules. For a ring $A$, a formal $A$-module is a formal group together with an action of the ring $A$ by endomorphisms of formal groups. There is a Hopf algebroid $\left(L_{A}, W_{A}\right)$ representing formal $A$-modules and strict isomorphisms between them. A formal group is a formal $\mathbb{Z}$-module. These Hopf algebroids are functorial in $A$.

Received by the editors September 25, 2005.

2000 Mathematics Subject Classification. Primary 55T25; Secondary 55N22, 14L05.

The author was supported in part by the NSF.

(C)2006 American Mathematical Society Reverts to public domain 28 years from publication 
If $A$ is the ring of integers in an algebraic extension of $\mathbb{Q}$, we get a map of Ext-groups

$$
\operatorname{Ext}_{(L, W)}^{* *}(L, L) \rightarrow \operatorname{Ext}_{\left(L_{A}, W_{A}\right)}^{* *}\left(L_{A}, L_{A}\right) .
$$

The domain of this map is the $E_{2}$-term of the Adams-Novikov spectral sequence for the homotopy groups of the sphere, while the range is, by definition, the AdamsNovikov $E_{2}$-term for formal $A$-modules. It is natural to ask whether this map of spectral sequences arises as a filtration of a map $\mathbb{S} \rightarrow \mathbb{S}_{A}$ from the sphere spectrum to an algebraic extension of $\mathbb{S}$.

Unfortunately, the answer is no in general. If $A=\mathbb{Z}[i]$, we will indicate as an example a computation of the first few terms of the 2-primary formal $\mathbb{Z}[i]$-module Adams-Novikov spectral sequence in section 3, together with the map from the ordinary Adams-Novikov sequence. This map would violate the nontrivial extension in the 3-stem. (A rough calculation seems to indicate that for an extension field totally ramified at an odd prime, the existence of the Toda differential does not immediately give rise to a contradiction.)

The extension in the 3-stem is detected by the ordinary Adams spectral sequence, suggesting that there is some incompatibility with the Steenrod algebra. By making this incompatibility precise, we find that the following general result holds.

Theorem 1.1. Let $A$ be the ring of integers in a finite extension field of $\mathbb{Q}$. There is no diagram of $E_{\infty}$-ring spectra

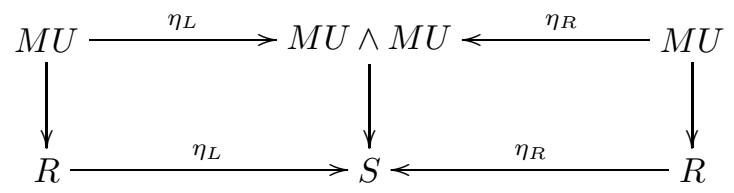

realizing the diagram

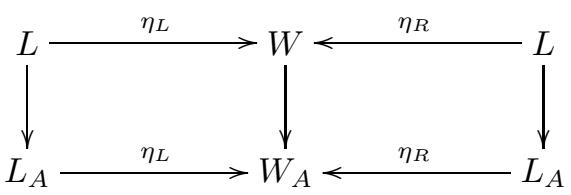

on homotopy groups unless $A=\mathbb{Z}$.

It follows from this that there exists no $E_{\infty}$ "algebraic sphere" $\mathbb{S}_{A}$ such that $\pi_{*}\left(\mathbb{S}_{A} \wedge M U\right) \cong L_{A}$ as an $L$-algebra. Given such an $\mathbb{S}_{A}$, an explicit computation with the Künneth spectral sequence for

$$
\mathbb{S}_{A} \wedge M U \wedge M U \simeq\left(\mathbb{S}_{A} \wedge M U\right) \underset{M U}{\wedge}(M U \wedge M U),
$$

together with unit maps arising from the weak equivalence

$$
\left(\mathbb{S}_{A} \wedge M U\right) \underset{\mathbb{S}_{A}}{\wedge}\left(\mathbb{S}_{A} \wedge M U\right) \rightarrow \mathbb{S}_{A} \wedge M U \wedge M U,
$$

would show that the pair $\left(\mathbb{S}_{A} \wedge M U, \mathbb{S}_{A} \wedge M U \wedge M U\right)$ violates Theorem 1.1.

As a partial converse, if we further assume that $(R, S)$ forms a Hopf algebroid of spectra (having comultiplication and augmentation maps that satisfy appropriate diagrams, in addition to the given left and right units), the algebraic sphere $\mathbb{S}_{A}$ could be recovered by the cobar construction $C(R, S, R)$. (This follows because the 
natural map $R \wedge M U \rightarrow S$ is a weak equivalence, and the smash product with $M U$ can be moved inside the cobar construction.)

The proof of Theorem 1.1 proceeds by calculating what the analog of the dual Steenrod algebra would be. This proof occupies section 4. In fact, the theorem holds locally at any prime of $A$ whose decomposition group is nontrivial; this is a phenomenon associated to any extension of the local field, rather than merely to the ramified primes.

The author would like to thank Michael Hill and Haynes Miller for discussions related to this note.

\section{The LaZArd Ring For Formal $A$-MOdules}

In this section, we will make explicit the form of the Lazard ring for formal $A$-modules for specific choices of $A$. The formulas from this section are taken from Hazewinkel [3], section IV.21.

If $A$ is a ring, a formal $A$-module is a formal group law $F$ over an $A$-algebra $R$ with a map $\phi: A \rightarrow \operatorname{End}(F)$ such that the diagram

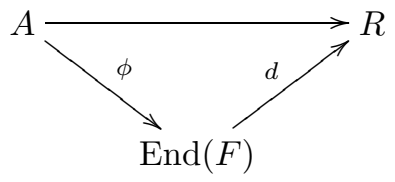

commutes, where $d$ is the differential at 0. For $a \in A$, the endomorphism $\phi(a)$ of $F$ is a power series $[a](X) \in R[[X]]$.

We now restrict to a particular case. Let $K$ be a finite extension of $\mathbb{Q}_{p}$ of degree $n$, with ring of integers $A$. Choose a uniformizer $\pi$ of $A$. As ideals of $A,(p)=\left(\pi^{e}\right)$, where $e$ is the ramification index. The residue field $A /(\pi)$ has order $q=p^{f}$. These satisfy $e \cdot f=n$.

For a positive integer $n$, define $\nu(n)=1$ if $n$ is not a prime power and $\ell$ if $n$ is a power of some prime $\ell$. Finally, let $C_{n}(X, Y)=\nu(n)^{-1}\left((X+Y)^{n}-X^{n}-Y^{n}\right)$.

Define $L_{A}$ to be the Lazard ring for formal $A$-modules, and $F_{A}$ the universal formal $A$-module over $L_{A}$. As a ring,

$$
L_{A} \cong A\left[Y_{1}, Y_{2}, Y_{3}, \ldots\right] .
$$

The coefficients can be identified. Modulo $Y_{1}, \ldots, Y_{n-2}$ and terms of degree $n+1$ and higher in $X$ and $Y$, we find the following:

$$
F_{A}(X, Y) \equiv \begin{cases}X+Y+Y_{n-1} \nu(n) C_{n}(X, Y) & \text { if } n \neq q^{m} \\ X+Y+\pi^{-1} Y_{n-1} \nu(n) C_{n}(X, Y) & \text { if } n=q^{m}\end{cases}
$$

(3, IV.21.4.8).

By restriction, a formal $A$-module is also a formal group law, and this corresponds to a map $L \rightarrow L_{A}$. If $F$ is the universal formal group law over $\mathbb{Z}$, we know that $L \cong \mathbb{Z}\left[X_{1}, X_{2}, \ldots\right]$. Modulo $X_{1}, \ldots, X_{n-2}$ and terms of degree $n+1$ and higher,

$$
F(X, Y) \equiv X+Y+X_{n-1} C_{n}(X, Y) .
$$


Therefore, the map $L \rightarrow L_{A}$ can be expressed as follows:

$$
X_{n} \mapsto \begin{cases}Y_{n}, & n \neq p^{m}-1, \\ p Y_{n}, & n=p^{m}-1, n \neq q^{k}-1, \\ \left(\frac{p}{\pi}\right) Y_{n}, & n=q^{k}-1 .\end{cases}
$$

The ring $L_{A}$ is part of a Hopf algebroid $\left(L_{A}, W_{A}\right)$ representing formal $A$-modules and strict isomorphisms between them. There are left and right unit maps $\eta_{L}, \eta_{R}$ : $L_{A} \rightarrow W_{A}$. As a module over $\eta_{L}\left(L_{A}\right)$,

$$
W_{A} \cong L_{A}\left[b_{1}, b_{2}, \ldots\right] \text {. }
$$

We choose the coefficients $b_{i}$ to be the coefficients of the universal strict isomorphism

$$
f(x)=x+\sum b_{i} x^{i+1}
$$

from $f^{-1} \circ F_{A} \circ f$ to $F_{A}$.

If $(L, W)$ is the Hopf algebroid representing formal groups and strict isomorphisms, we know that

$$
W \cong L\left[b_{1}, b_{2}, \ldots\right]
$$

as a module over $\eta_{L}(L)$. The restriction map $L \rightarrow L_{A}$ extends to a map $(L, W) \rightarrow$ $\left(L_{A}, W_{A}\right)$ of Hopf algebroids. The map $W \rightarrow W_{A}$ is the extension of scalars map $L\left[b_{i}\right] \rightarrow L_{A}\left[b_{i}\right]$.

The ring $W_{A}$ has a quotient ring $A \otimes_{L_{A}} W_{A}$, where the map $L_{A} \rightarrow A$ classifies the additive group law. This ring, which is isomorphic to $A\left[b_{1}, b_{2}, \ldots\right]$, classifies the universal strict isomorphism whose range is the additive formal group law. We now wish to determine the image of the right unit, which is equivalent to determining the domain formal group law.

Proposition 2.1. Modulo $\eta_{R}\left(Y_{1}\right), \ldots, \eta_{R}\left(Y_{n-1}\right)$, the image of $\eta_{R}\left(Y_{n}\right)$ in the ring $A\left[b_{1}, b_{2}, \ldots\right]$ is $-b_{n}$ if $n \neq q^{m}-1$ for any $m$, and $-\pi b_{n}$ if $n=q^{m}-1$.

Proof. If $f(x)=x+\sum b_{i} x^{i+1}$ is the universal strict isomorphism, then $f$ is a map from $G$ to the additive formal group law, where $G(x, y)=f^{-1}(f(x)+f(y))$. By equation (1), when we reduce modulo $\eta_{R}\left(Y_{1}\right), \ldots, \eta_{R}\left(Y_{n-2}\right)$ and terms of degree $n+1$ and higher, we find that

$$
f^{-1}(f(x)+f(y)) \equiv \begin{cases}X+Y+\eta_{R}\left(Y_{n-1}\right) \nu(n) C_{n}(X, Y) & \text { if } n \neq q^{m}, \\ X+Y+\eta_{R}\left(Y_{n-1}\right) \pi^{-1} \nu(n) C_{n}(X, Y) & \text { if } n=q^{m} .\end{cases}
$$

Applying $f$ to both sides, we find that

$$
f(x)+f(y) \equiv \begin{cases}f\left(X+Y+\eta_{R}\left(Y_{n-1}\right) \nu(n) C_{n}(X, Y)\right) & \text { if } n \neq q^{m}, \\ f\left(X+Y+\eta_{R}\left(Y_{n-1}\right) \pi^{-1} \nu(n) C_{n}(X, Y)\right) & \text { if } n=q^{m} .\end{cases}
$$

Taking terms of degree $n$ gives the formula

$$
b_{n-1} X^{n}+b_{n-1} Y^{n} \equiv \begin{cases}\eta_{R}\left(Y_{n-1}\right) \nu(n) C_{n}(X, Y)+b_{n-1}(X+Y)^{n} & \text { if } n \neq q^{m} \\ \eta_{R}\left(Y_{n-1}\right) \pi^{-1} \nu(n) C_{n}(X, Y)+b_{n-1}(X+Y)^{n} & \text { if } n=q^{m}\end{cases}
$$

In particular, modulo $\eta_{R}\left(Y_{1}\right), \ldots, \eta_{R}\left(Y_{n-2}\right)$, we have $\eta_{R}\left(Y_{n-1}\right) \equiv-b_{n-1}$ if $n \neq$ $q^{m}$, and $\eta_{R}\left(Y_{n-1}\right) \equiv-\pi b_{n-1}$ if $n=q^{m}$. Re-indexing gives the statement of the proposition. 


\section{The formal $\mathbb{Z}_{2}[i]$-Module Adams-Novikov Sequence}

In this section, we fix $A$ to be the ring $\mathbb{Z}_{2}[i]$. We will compute the formal $A$ module Adams-Novikov spectral sequence out to dimension 3 by using the reduced bar complex

$$
0 \rightarrow L_{A} \rightarrow \bar{W}_{A} \rightarrow \bar{W}_{A} \otimes_{L_{A}} \bar{W}_{A} \rightarrow \cdots,
$$

where $\bar{W}_{A}$ is the kernel of the augmentation map $\epsilon: W_{A} \rightarrow L_{A}$.

Let $\pi=1+i$ be a uniformizer for $A$. We can choose generators of $L_{A}$ and $W_{A}$, as in section 2] such that $X_{1} \mapsto \pi Y_{1}$ and $b_{1} \mapsto b_{1}$. As $\eta_{R} X_{1}=X_{1}-2 b_{1}$, we deduce the formula $\eta_{R}\left(Y_{1}\right)=Y_{1}-(2-\pi) b_{1}$. Similarly, $\Delta b_{1}=1 \otimes b_{1}+b_{1} \otimes 1$. These formulas allow us to directly compute the homology of this complex out to the 3-stem; we record the result in the following diagram. The vertical axis denotes Ext-degree, and the horizontal axis denotes total degree.

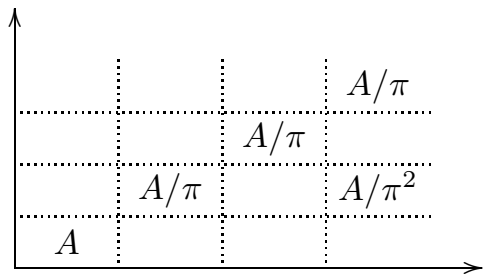

The elements in total degree 3 are generated by the cycles $\nu_{A}=b_{1}^{2}-\pi Y_{1} b_{1}$ and $\eta_{A}^{3}=b_{1} \otimes b_{1} \otimes b_{1}$ in the bar complex. The map of Adams-Novikov sequences sends the element $\nu=b_{1}^{2}-X_{1} b_{1}$ to $\nu_{A}$, and sends $\eta^{3}$ to $\eta_{A}^{3}$.

This violates the extension in the 2-primary Adams-Novikov spectral sequence. A lift of $\nu$ to $\pi_{3}(\mathbb{S})$ satisfies $4 \nu=\eta^{3}$, but any lifting of $\nu_{A}$ would have to be $\pi^{3}$ torsion, and hence be killed by $\pi^{4}=-4$.

\section{The formal $A$-module Steenrod Algebra}

We now prove the following. Let $A$ be the ring of integers in a finite extension of $\mathbb{Q}_{p}$, and let $\left(L_{A}, W_{A}\right)$ be the Hopf algebroid associated to $A$.

Theorem 4.1. There is no diagram of $E_{\infty}$-ring spectra

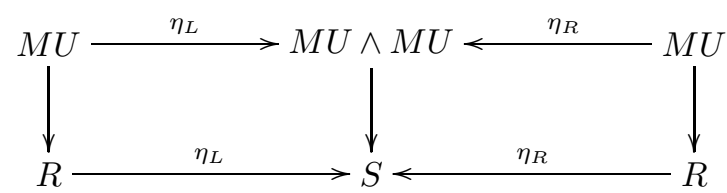

realizing the diagram

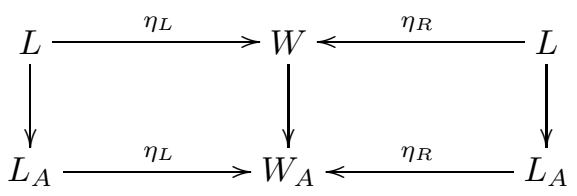

on homotopy groups unless $A=\mathbb{Z}_{p}$.

Theorem 1.1 follows immediately by completing at any prime that is not totally split. 
Proof. Suppose that we had a diagram of $E_{\infty}$-ring spectra as stated. We have that $R$ is (-1)-connected with $\pi_{0} R=A$, so we can form a diagram of $E_{\infty}$-ring spectra

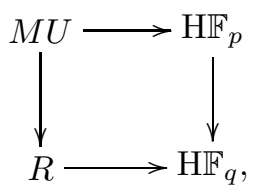

where $\mathbb{F}_{q}$ is the residue field of $A$.

We then get a map of $E_{\infty}$-ring spectra

$$
\mathrm{HF}_{p} \underset{M U}{\wedge}(M U \wedge M U) \rightarrow \mathrm{HF}_{q} \underset{R}{\wedge} S
$$

where the smash product is taken along the left unit. The Künneth spectral sequence of [2] shows that on homotopy groups, this map is the extension of scalars map

$$
\mathbb{F}_{p}\left[b_{1}, b_{2}, \ldots\right] \rightarrow \mathbb{F}_{q}\left[b_{1}, b_{2}, \ldots\right] .
$$

These rings respectively classify the universal strict isomorphisms into the additive formal groups over $\mathbb{F}_{p}$ and $\mathbb{F}_{q}$.

Smashing along the right unit then gives a map of $E_{\infty}$-ring spectra

$$
\mathrm{HF}_{p} \underset{M U}{\wedge}(M U \wedge M U) \underset{M U}{\wedge} \mathrm{HF}_{p} \rightarrow \mathrm{HF}_{q} \underset{R}{\wedge} S \underset{R}{\wedge} \mathrm{HF}_{q}
$$

The homotopy groups of the left-hand spectrum form the dual Steenrod algebra $\mathcal{A}_{*}$. Write $\mathcal{B}_{*}=\pi_{*}\left(\mathrm{HF}_{q} \wedge_{R} S \wedge_{R} \mathrm{HF}_{q}\right)$.

We get by naturality a map of Künneth spectral sequences

$$
\operatorname{Tor}_{* *}^{L}\left(\mathbb{F}_{p}\left[b_{i}\right], \mathbb{F}_{p}\right) \rightarrow \operatorname{Tor}_{* *}^{L_{A}}\left(\mathbb{F}_{q}\left[b_{i}\right], \mathbb{F}_{q}\right)
$$

that converges to some filtration of the map $\mathcal{A}_{*} \rightarrow \mathcal{B}_{*}$.

Proposition 2.1 shows that the image of $\eta_{R}\left(Y_{i}\right)$ in $\mathbb{F}_{q}\left[b_{1}, b_{2}, \ldots\right]$ is congruent modulo $\eta_{R}\left(Y_{1}\right), \ldots, \eta_{R}\left(Y_{i-1}\right)$ to $-b_{i}$ if $i \neq q^{m}-1$, and 0 if $i=q^{m}-1$. The same proposition shows that a similar result holds for $X_{i}$ with $q$ replaced by $p$. This determines the right module structure of $\mathbb{F}_{p}\left[b_{i}\right]$ and $\mathbb{F}_{q}\left[b_{i}\right]$.

The sequence $\left(p, X_{1}, X_{2}, \ldots\right)$ is regular in $L$, so we can resolve $\mathbb{F}_{p}$ over $L$ by the Koszul complex

$$
\bigotimes_{i=0}^{\infty}\left(L \stackrel{X_{i}}{\longrightarrow} L\right)
$$

where $X_{0}=p$ by convention. The tensor product is taken over $L$. Similarly, $\left(\pi, Y_{1}, Y_{2}, \ldots\right)$ is regular in $L_{A}$, so we have a similar Koszul resolution for $\mathbb{F}_{q}$ over $L_{A}$.

The following Tor calculations follow:

$$
\begin{aligned}
\operatorname{Tor}_{* *}^{L}\left(\mathbb{F}_{p}\left[b_{i}\right], \mathbb{F}_{p}\right) & \cong \mathbb{F}_{p}\left[\xi_{1}, \xi_{2}, \ldots\right] \otimes \Lambda\left[\tau_{0}, \tau_{1}, \tau_{2}, \ldots\right], \\
\operatorname{Tor}_{* *}^{L_{A}}\left(\mathbb{F}_{q}\left[b_{i}\right], \mathbb{F}_{q}\right) & \cong \mathbb{F}_{q}\left[\xi_{f}, \xi_{2 f} \ldots\right] \otimes \Lambda\left[\sigma_{0}, \sigma_{f}, \sigma_{2 f}, \ldots\right] .
\end{aligned}
$$

The elements $\xi_{k}$ live in Tor degree zero and total degree $2\left(p^{k}-1\right)$. (The element $\xi_{k}$ is represented by $b_{p^{k}-1}$.) The elements $\tau_{k}$ live in Tor degree 1 and total degree $2 p^{k}-1$, while the elements $\sigma_{k f}$ live in Tor degree 1 and total degree $2 q^{k}-1$. Because the terms in the spectral sequence are generated by terms in homological degrees 0 and 1 , the spectral sequence collapses. 
The map of spectral sequences sends $\xi_{k}$ to 0 if $f$ does not divide $k$. In fact, the Tor-degree zero terms form the algebras $\mathbb{F}_{p} \otimes_{L} W \otimes_{L} \mathbb{F}_{p}$ and $\mathbb{F}_{q} \otimes_{L_{A}} W_{A} \otimes_{L_{A}} \mathbb{F}_{q}$ respectively. These rings classify the strict automorphisms of the additive formal group laws over $\mathbb{F}_{p}$ and $\mathbb{F}_{q}$. As such, they are the affine coordinate rings of the group schemes of $p$-series and $q$-series respectively.

The map of Tor-groups is induced by a map of Koszul resolutions; this map of resolutions is formed by tensoring together the maps

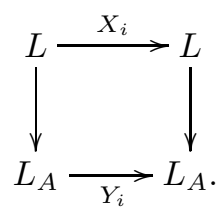

The right vertical map is the natural inclusion. In order to make the diagram commute, the left vertical map must be the inclusion if $i \neq p^{m}-1$, multiplication by $p$ if $i=p^{m}-1, i \neq q^{k}-1$ for any $k$, and multiplication by $\pi$ if $i=q^{k}-1$, by equation (2).

We then carry forward the computation on Tor. We find that the image of $\tau_{k f}$ is $\sigma_{k f}$ if the extension is unramified. In any other case, $\tau_{k}$ maps to zero.

However, there can be no such map $\mathcal{A}_{*} \rightarrow \mathcal{B}_{*}$ that respects the $E_{\infty}$-structure unless the field extension is trivial. The reason is as follows.

The element $\xi_{f}$ in $\mathcal{A}_{*}$ would have nonzero image in $\mathcal{B}_{*}$, and hence so does its conjugate $\chi \xi_{f}$. The field extension is nontrivial, so either $f>1$ (implying that $\mathcal{B}_{*}$ is trivial in dimensions 2 through $2 q-3$ ), or the extension is totally ramified and $\tau_{0}$ maps to 0 . In either case, $\chi \tau_{f-1}$ maps to 0 .

If $p=2$, this is immediately a contradiction, as $\chi \tau_{f-1}$ lifts to a class in $\mathcal{A}_{*}$ that squares to a nonzero multiple of $\chi \xi_{f}$.

If $p>2$, the map $\mathcal{A}_{*} \rightarrow \mathcal{B}_{*}$ is a map of $E_{\infty}$-algebras over $\mathrm{HF}_{p}$, and so it should respect the Dyer-Lashof operations. However, $\beta Q^{p^{k}} \chi \tau_{f-1}=\chi \xi_{f}$ in $\mathcal{A}_{*}$ ([1], Theorem III.2.3). The element $\chi \tau_{f-1}$ has trivial image and $\chi \xi_{f}$ has nontrivial image, which gives a contradiction.

Remark 4.2. For clarity, we have chosen to assume that the object $S$ is an $E_{\infty}$ ring spectrum; it is possible to weaken this assumption. There is a folklore result (for which the author does not know of a reference in the literature) to the effect that $\chi \xi_{f}=\beta Q^{p^{k}}\left(\chi \tau_{f-1}\right)$ can be identified with the $p$-fold Massey product $\left\langle\chi \tau_{f-1}, \ldots, \chi \tau_{f-1}\right\rangle$. The fact that the map $\mathcal{A}_{*} \rightarrow \mathcal{B}_{*}$ gives rise to a contradiction only depends on the existence of $p$-fold Massey products, and these are well-defined for $A_{p}$-algebras. We could therefore restrict our assumptions to $S$ being an $A_{p^{-}}$ algebra over $R \wedge R^{o p}$.

\section{REFERENCES}

1. R. R. Bruner, J. P. May, J. E. McClure, and M. Steinberger, $H_{\infty}$ ring spectra and their applications, Lecture Notes in Mathematics, vol. 1176, Springer-Verlag, Berlin, 1986. MR0836132 (88e:55001)

2. A. D. Elmendorf, I. Kriz, M. A. Mandell, and J. P. May, Rings, modules, and algebras in stable homotopy theory, Mathematical Surveys and Monographs, vol. 47, American Mathematical Society, Providence, RI, 1997, with an appendix by M. Cole. MR.1417719 (97h:55006) 
3. Michiel Hazewinkel, Formal groups and applications, Pure and Applied Mathematics, vol. 78, Academic Press Inc. [Harcourt Brace Jovanovich Publishers], New York, 1978. MR0506881 (82a:14020)

4. Daniel Quillen, On the formal group laws of unoriented and complex cobordism theory, Bull. Amer. Math. Soc. 75 (1969), 1293-1298. MR0253350 (40:6565)

5. Douglas C. Ravenel, Formal A-modules and the Adams-Novikov spectral sequence, J. Pure Appl. Algebra 32 (1984), no. 3, 327-345. MR0745362 (86a:55023)

6. John Rognes, Galois extensions of structured ring spectra, Available online at http://www.math.uio.no/ rognes/publications.html.

7. R. Schwänzl, R. M. Vogt, and F. Waldhausen, Adjoining roots of unity to $E_{\infty}$ ring spectra in good cases - a remark, Homotopy invariant algebraic structures (Baltimore, MD, 1998), Contemp. Math., vol. 239, Amer. Math. Soc., Providence, RI, 1999, pp. 245-249. MR1718085 (2001b:55026)

Department of Mathematics, Massachusetts institute of Technology, Cambridge, MassachusetTS 02139

E-mail address: tlawson@math.mit.edu 